\title{
Water Quality Evaluation in Term of WQI River Tungabhadra, Karnataka, India.
}

\author{
S.Ranjith, Anand.V. Shivapur, P. Shiva Keshava Kumar, Chandrashekarayya.G. Hiremath, \\ Santhosh Dhungana
}

\begin{abstract}
The study reports the Weighted Arithmetic Water Quality Index $\left(W Q I_{a}\right)$ value obtained for River Tungabhadra, a major tributary of Krishna River basin. A WQI ${ }_{a}$ delivers a unique rating that gives whole water quality at a specific stretch and period depending upon some water quality constraints. The principle point of a $\mathrm{WQI}_{a}$ is to give complex water quality insights into data that is clear and useable by the community. Some of most critical water quality parameters such as $\mathrm{pH}$, Total dissolved solids (TDS), Total alkalinity, dissolved oxygen (DO), Biochemical oxygen demand (BOD), Total hardness (TH), calcium (Ca), magnesium (Mg), and electrical conductivity $(\mathrm{EC})$ were Used for evaluating the $\mathrm{WQI}_{a}$. The $\mathrm{WQI}_{a}$ esteems for the Tungabhadra River oscillate from 40 to 156. The estimations of $\mathrm{WQI}_{a}$ exhibited that the stream water was free of any impurities at the examining sites aside from 2-3 months where its qualities were under good condtion. On every occasion there are anthropogenic influence viz industrial effluent, agricultural runoff and domestic sewage which is directly discharge into stream water gets contaminated to some level and hence of WQI declines. It is opinioned that $W Q I_{a}$ can be used as a device in relating the water-quality of different sources. It delivers the community a over-all awareness of the thinkable glitches with water in a specific stretch. The WQI are among the best approaches to convey the data on water-quality pattern to the public community or to the water quality policy-makers and which is help full to drive suitable mitigative measure.
\end{abstract}

Rundown phrases- water-quality parameter, weighted arithmetic water quality index (WQI), Tungabhadra River.

\section{I.INTRODUCTION}

The Tungabhadra River is a familiar river in South India and is almost travels $531 \mathrm{~km}$ in distance. The Tungabhadra stream starts its ride from Koodli of Shivamoga district in the Karnataka state of India. The majority course of the stream flows in the state of Karnataka, India.

$\mathrm{WQI}_{\mathrm{a}}$ is a rating system to delineate the general water-quality grade in a stretch that is useful for the choice of suitable treatment procedure to meet the concerned problems (Tyagi et al.2013). WQI is a tool to decide state of water quality of stream. Calculating the WQI contains three stages (US EPA 2009): (i) in that initial step which acquire measurements on separate water quality constraint, (ii) convert into "sub index" values (iii) finally total the individual sub index into general water quality index esteem.

Revised Manuscript Received on July 18, 2019. India.

S.Ranjith, Research Student in VTU-PG studies, Belagavi-590018,

Dr. Anand.V. Shivapur, Regional Director and PG-coordinator VTUPG studies, Belagavi-590018, India.

Dr. P. Shiva Keshava Kumar, Professor and Head, Department of Civil Engineering, PDIT Engineering College Hosapete-583201, India.

Mr.Chandrashekarayya.G. Hiremath, Assistant prof. Department of water and land management, VTU, Belagavi-590018, India

Santhosh Dhungana, . program officer, School of Environment, Resources and Development (SERD), Pathumthani 12120, Thailand.
Series of mathematical equations mostly based on the mean methods like arithmetic, harmonic, logarithmic, mean multiplicative are widely adopted by various authors in developing water quality indices.

In 1965 R.K.Horton develop a water quality index based on aggregation arithmetic function by selecting ten most frequently parameter for $\mathrm{WQI}_{\mathrm{a}}$ which include Biochemical oxygen demand (BOD),

dissolved oxygen (DO), $\mathrm{pH}$, coliforms, Total suspended and Total dissolved Solids, Total alkalinity, and chloride. The aggregation of arithmetic considering of the water quality factor was replicated with the temperature and "evident contamination" to get the total capacity from which the general WQI ${ }_{a}$ was discovered. The arithmetic $\mathrm{WQI}_{\mathrm{a}}$ weight fluctuated from one to four. Likewise, R.K. Horton.et.al. (1965), Despite the importance of multiplication factor in arithmetic weighting, R. M. Brown. et al. (1970) did not consider it rather used essential number arithmetic weighting National sanitation foundation (NSF) develop a water quality index based on Delphi technique which is expliained in Dalkey 1968, in that logarithmic function to adapt water quality factor consequences hooked on sub-index values.

According to S.H.Dinius in 1987 develop a $\mathrm{WQI}_{\mathrm{m}}$ model based on mean multiplicative having decrease rating scale, with qualities communicated as a level of clean water quality relating to $100 \%$. Similar effort was attempted by Rescher and Helmer 1959, Helmer and Dalkey 1963 by presenting changes to Delphi method (Dalkey 1968). Brown et al. (1972), Bhargava et al. (1998), Dwivedi et al. (1997), Deininger and Landwehr (1976) provided multiplicative form of the index where weights to individual parameters were assigned based on a subjective opinion on the judgment and critical analysis of the author. N.Dee et al. (1973) anticipated a structure for assessing ecological factor effect of significant water-resources schemes.

McClelland (1974) developed a geometric function to estimate the WQI $\mathrm{I}_{\mathrm{g}}$. He was of the opinion that the number arithmetic mean needed Affectability to low esteem parameters, a trademark later considered "covering". He as an alternative proposed the "weighted geometric mean". Procedure latter (Deininger and Landwehr 1976; Parker and Walski 1974; Bhargava et al.1983; S H Dinius 1987) have additionally utilized a weighted geometric mean for aggregation.

Dojlido et al. 1994 Utilize the harmonic mean to discover the $\mathrm{WQI}_{\mathrm{h}}$. This mean does not utilize weights for the individual parameter indicator. he is found that it was more

Published By

Blue Eyes Intelligence Engineering

\& Sciences Publication

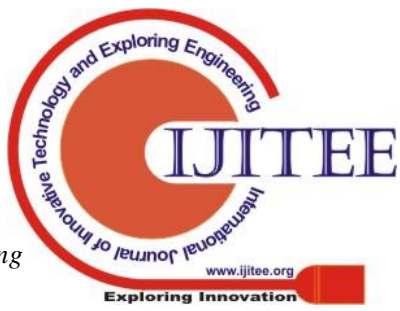


delicate to the most disabled marker than the numbercrunching or symphonious methods, decreasing overshadowing, while as yet representing the impact of different pointers (Walsh and Wheeler 2012). The either methods used are "Canadian Council of Ministers of the Environment Water Quality Index (CCMEWQI) and British Columbia water quality Index". The "Canadian Council of Ministers of the Environment Water Quality Index" analyzes perceptions to a benchmark as opposed to normalizing watched qualities to abstract score curves, where the benchmark might be a water quality standard or site explicit foundation focus (CCME 2001; Khan et al. 2003; Lumb et al. 2006). "British Columbia water quality index" was created by the Canadian Ministry of Environment in 1995 as expanding file to assess water quality. This file is like CCME-WQI where water quality parameters are estimated and their infringement is controlled by examination with a predefined limit. It gives probability to make a characterization based on all current estimation parameters (Bharti and Katyal 2011).

Smith et al. (1987a, b) formulated an index based on optimized operator for four different class of water utilization i.e., point and non-point. It is a cross breed of the two regular index types and is depend on professional judgement as well as water-quality values. The selection of constraints for each water class, evolving sub-indices, and conveying weightages were all done using Delphi. The minimum operator technique was used to obtain the final index score (Bharti and Katyal 2011):

$\mathrm{I}_{\min }=\sum \operatorname{Min}(\mathrm{I}$ sub1, I sub2,...,)

$\operatorname{Imin}=$ the lowermost sub-index rate.

In over-all, WQI are parted into five steps (Sobhani 2003):

A) Community/public indices: this method assesses the water quality index neglects the application of water use for consumptions like drinking, irrigation, industrialization and aqua culture etc.,

B) Exact/specific consumption indices: aforementioned water uses application-based categorization of the water quality.

C) Statistical indices: indices are derived based on numerical/ statistical methods involving equations rather than the individual opinion.

D) Designing indices: decision support indices for preparation water quality managing the projects.

In this research work, the "weighted arithmetic mean" $\left(\mathrm{WQI}_{\mathrm{a}}\right)$ technique is used.

$$
W Q I a=\sum_{i=1}^{n} q i * W \mathrm{i}
$$

$\mathrm{W}_{\mathrm{i}}=1$ where $\mathrm{W}_{\mathrm{i}}$ is the unit weight of each constraint, $\mathrm{q}_{\mathrm{i}}=$ sub-index rating for each variable and $n$ is the number of sub-indices aggregated.

The benefits of $\mathrm{WQI}_{\mathrm{a}}$ method used in this study are (Tyagi et al. 2013).

1. This technique includes data from several water quality parameter into a scientific calculation that rates the healthiness of water body with number.

2. Data light assessment method meaning reduced number of variables requirement compared to all other water quality variables for specific use.
3. Useful method for assessment of water quality as it incorporates the weighted influence of the variables involved and assists decision makers in adopting appropriate strategies to cope with it

Replicates the compound effect of diverse varibles i.e., significant for the valuation and managing of stream water quality.

\section{MATERIAL AND METHODOLOGY}

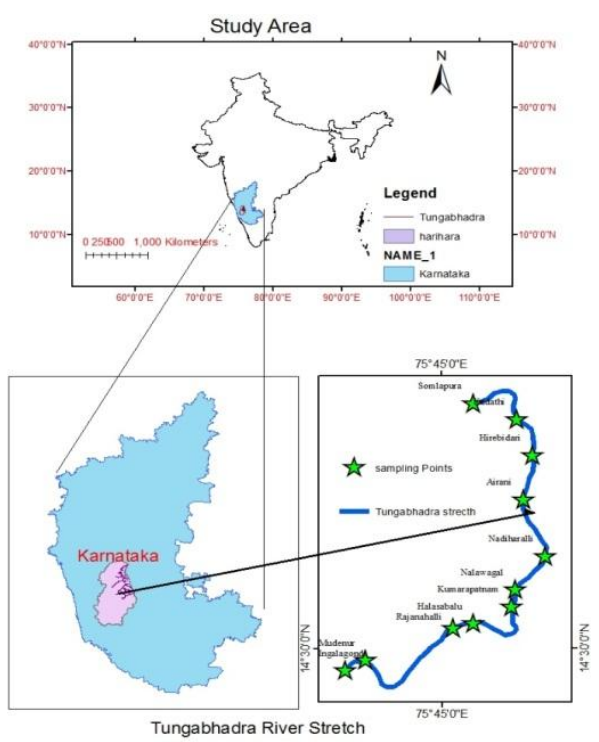

Fig.1 location of sampling station.

Stream water samples were Collected. from seven sampling-station viz. Mudenuru (SS1), Rajanahalli (SS2), Kumarapatnam bridge (SS3), Nalawagal (SS4), Nadhiharalli (SS5), Airani (SS6) and Heribedri (SS7) during postmonsoon (POM), Pre-monsoon (PRM) and Monsoon (POM) season over a period of twelve month, i.e., from January 2018 to December 2018. The particulars of sampler station are shown in Fig. 1.

Several physico-chemical and biological constraints of the water samples were examined by following the typical organizations of APHA (2005). A set of ten most regularly used water quality parameters namely Electrical conductivity (EC), Total dissolved solid (TDS), Total suspended solid (TSS), pH, Total alkalinity (TA), Total hardness (TH), Chloride, Dissolved oxygen (DO), Biochemical oxygen demand (BOD) and sulphate which, organized, reflect the complete water quality of the Tungabhadra River were chosen for producing the $\mathrm{WQI}_{\mathrm{a}}$ was eastimated by following the "weighted arithmetic index method" (Brown et al. 1970), using the condition:

$\mathrm{WQI}=\sum \mathrm{Q}_{\mathrm{n}} \mathrm{W}_{\mathrm{n}} / \sum \mathrm{W}_{\mathrm{n}}$

Where

$\mathrm{Q}_{\mathrm{n}}=$ quality rating is designed using thes condition $\mathrm{Qn}=100[(\mathrm{Vn}-\mathrm{Vi}) /(\mathrm{Vs}-\mathrm{Vi})] \mathrm{Qn}=100[(\mathrm{Vn}-\mathrm{Vi}) /(\mathrm{Vs}-\mathrm{Vi})]$

$V_{n}=$ actual amount of $n$th parameter present, $V_{i}$ is the ideal value of the parameter $\left[V_{i}=0\right.$, except for $\mathrm{pH}\left(V_{i}=7\right)$ 
and DO $\left.\left(V_{i}=14.6 \mathrm{mg} / \mathrm{l}\right)\right], V_{s}$ is the standard permissible value for the $n$th water quality parameterUnit weight $\left(W_{n}\right)$ is calculated using the formula

$\mathrm{Wn}=\mathrm{k} / \mathrm{Vs}$ where $k=$ proportionality constant i.e., $\mathrm{k}=\left[1 / \sum 1 / \mathrm{Vs}=1,2, \ldots, \mathrm{n}\right]$ (Brown et al. 1970).

\section{RESULTS AND DISCUSSION}

For ascertaining Water quality index, the prime preessential is the consequences of different water quality parameter analysis. The numerical summary of the chose water quality parameters at various sampling station of the Tungabhadra River all over Pre-monsoon, Monsoon and Post monsoon season is displayed in Table 2.

Table 1 water quality range and possible usage of water sample (brown et al. 1970)

\begin{tabular}{cll}
\hline WQI & Water quality status & \multicolumn{1}{c}{ Possible usage } \\
\hline $0.0-25.0$ & Excellent. & Drinking, irrigation and industrial. \\
$26.0-50.0$ & Good. & Drinking, irrigation and industrial. \\
$51.0-75.0$ & Poor. & Irrigation and industrial. \\
$76.0-100.0$ & very poor. & Irrigation. \\
$>100.0$ & unsuitable for drinking and & Proper treatment required before use. \\
& aquaculture. & \\
\hline
\end{tabular}

Table 2. Range of value for water quality parameter of Tungabhadra river.

\begin{tabular}{llll}
\hline Parameter & \multicolumn{1}{c}{ Pre-monsoon } & \multicolumn{1}{c}{ Monsoon } & \multicolumn{1}{c}{ Post-monsoon } \\
\hline Ph & $7.46 \pm 0.607(6.7-8.25)$ & $7.49 \pm 0.476(6.8-8.2)$ & $7.5 \pm 0.50(6.85-7.85)$ \\
EC & $350.3 \pm 74.2(221-440)$ & $222.23 \pm 36.9(155-240)$ & $218 \pm 34.99(185.5-285.5)$ \\
TDS & $328.55 \pm 97.96(182-440)$ & $129.7 \pm 20.3(91-140)$ & $137.5 \pm 25.92(116.5-195.5)$ \\
TSS & $190.42 \pm 120.262(60-385)$ & $222.15 \pm 131.52(75-375)$ & $190.428 \pm 120(62-185.5)$ \\
TH & $134.42 \pm 29.27(79.5-160.33)$ & $72.2 \pm 10.37(56.3-83.5)$ & $96.25 \pm 41.2(67-120.2)$ \\
chloride & $45.23 \pm 7.7(32.2-52.33)$ & $29.8 \pm 7.3(18.5-39.5)$ & $31.9 \pm 2.98(27.2-34.5)$ \\
DO & $6.8 \pm 1.2(5.1-8.4)$ & $8.12 \pm 1.02(6.3-9.1)$ & $7.36 \pm 0.86(6.01-8.2)$ \\
BOD & $4.77 \pm 2.57(2.2-6.84)$ & $3.96 \pm 1.75(2.05-5.3)$ & $3.59 \pm 2.136(2.4-8.1)$ \\
sulphate & $11.14 \pm 4.23(4.65-15)$ & $9.85 \pm 5.64(4.68-20.58)$ & $6.8 \pm 0.76(5.32-7.85)$ \\
TA & $142.23 \pm 29.5(81.34-164)$ & $96.83 \pm 22.11(64.8-112)$ & $96.302 \pm 35.3(60-170)$ \\
\hline \multicolumn{5}{c}{ Values expressed in mean with standard deviation (range bound) }
\end{tabular}

$\mathrm{pH}$ for the most part implies the level of acridity or alkalinity of a water. The normal $\mathrm{pH}$ esteems for premonsoon, monsoon and post-monsoon season were $7.45 \pm$ $0.60,7.42 \pm 0.40$ and $7.52 \pm 0.5$, respectively. Even with the fact that the normal $\mathrm{pH}$ range were within the BIS recommendation, in this case, sampling station 5 i.e Nalavagalu village having High $\mathrm{pH}$ around 8.3. Electrical conductivity dealings the electric current passing/carrying capacity of a river sample and which is linked to the total dissolved ions present in the stream water. Experiential Electrical conductivity values for the water sample of the Tungabhadra River fluctuated between 350.3 \pm 74.216 $\mu \mathrm{mho} / \mathrm{cm}, 222.3 \pm 36.914 \mu \mathrm{mho} / \mathrm{cm}$ and $218 \pm 35 \mu \mathrm{mho} / \mathrm{cm}$ over period Pre-monsoon, Monsoon and Post-monsoon season respectively, during Pre-monsoon season EC value exceed the BIS standard of $300 \mu \mathrm{mho} / \mathrm{cm}$ at some of the sampling station.
Table no.3 relative weight $W_{n}$ parameter used in $\mathrm{WQI}_{\mathrm{a}}$.

\begin{tabular}{lcc}
\hline Parameter & BIS Standard & Unit weight $\mathrm{W}_{\mathrm{n}}$ \\
\hline $\mathrm{pH}$ & $6.5-8.5$ & 0.215 \\
Eletrical Conductivity & 300 & 0.0061 \\
TDS & 500 & 0.00366 \\
TSS & 500 & 0.00366 \\
Total Hardness & 300 & 0.0061 \\
chloride & 250 & 0.00732 \\
DO & 5 & 0.366 \\
BOD & 5 & 0.366 \\
sulphate & 150 & 0.0122 \\
Total Alkalinity & 120 & 0.01525 \\
& $\Gamma \mathrm{W}_{\mathrm{n}}$ & 1.00129 \\
\hline
\end{tabular}

Table no .4 Values of water quality index at Sampling station 1.

\begin{tabular}{|c|c|c|c|c|c|c|c|c|c|}
\hline \multirow[b]{2}{*}{ Pafameter } & \multicolumn{3}{|c|}{ Pre-Monsoon } & \multicolumn{3}{|c|}{ Monsoon } & \multicolumn{3}{|c|}{ Post-Monsoon } \\
\hline & $V_{\text {In }}$ & QtI & 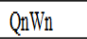 & $V_{11}$ & Q11 & $\mathrm{Qn}_{\mathrm{n}} \mathrm{Wn}_{\mathrm{n}}$ & $V_{n}$ & Q11 & $\mathrm{Qn}_{\mathrm{n} W \mathrm{n}}$ \\
\hline $\mathrm{pH}$ & 7.05 & 10 & 2.15 & 7.1 & 20 & 4.3 & 6.9 & -20 & -4.3 \\
\hline $\mathrm{EC}$ & 221.52 & 73.84 & 0.450424 & 155.2 & 51.73333 & 0.315573 & 187.9 & 62.63333 & 0.382063 \\
\hline TDS & 128.48 & 25.696 & 0.094047 & 91.58 & 18.316 & 0.067037 & 116.48 & 23.296 & 0.085263 \\
\hline TSS & 60 & 12 & 0.04392 & 75 & 15 & 0.0549 & 80 & 16 & 0.05856 \\
\hline $\mathrm{TH}$ & 79.45 & 26.48333 & 0.161548 & 56.9 & 18.96667 & 0.115697 & 67.7 & 22.56667 & 0.137657 \\
\hline chloride & 32.82 & 13.128 & 0.096097 & 18.48 & 7.392 & 0.054109 & 27.26 & 10.904 & 0.079817 \\
\hline DO & 8.4 & 64.58333 & 23.6375 & 9.1 & 57.29167 & 20.96875 & 7.59 & 73.02083 & 26.72563 \\
\hline $\mathrm{BOD}$ & 2.6 & 52 & 19.032 & 2.05 & 41 & 15.006 & 2.44 & 48.8 & 17.8608 \\
\hline sulphate & 6.896 & 4.597333 & 0.056087 & 4.78 & 3.186667 & 0.038877 & 5.32 & 3.546667 & 0.043269 \\
\hline \multirow[t]{3}{*}{$\mathrm{TA}$} & 135.4 & 1128333 & 1.720708 & 64.68 & 53.9 & 0.821975 & 72.38 & 60.31667 & 0.919829 \\
\hline & & $\sum$ WQI & 47.44233 & & $\sum$ WQI & 41.74292 & & $\sum$ WQI & 41.99288 \\
\hline & & WQI & 47.38121 & & WQI & 41.68914 & & WQI & 41.93878 \\
\hline
\end{tabular}

Table no .5 Values of water quality index at Sampling station 2.

\begin{tabular}{|c|c|c|c|c|c|c|c|c|c|}
\hline \multirow[b]{2}{*}{ Parameter } & \multicolumn{3}{|c|}{ Pre-Monsoon } & \multicolumn{3}{|c|}{ Monsoon } & \multicolumn{3}{|c|}{ Post-Monsoon } \\
\hline & $\mathrm{Vn}$ & Qn & $\mathrm{Qn}^{*} \mathrm{Wn}$ & Vn & Qn & $\mathrm{Q}{ }^{*} \mathrm{Wn}$ & $\mathrm{Vn}$ & Qn & $\mathrm{Qn} * \mathrm{Wn}$ \\
\hline $\mathrm{pH}$ & 7.14 & 28 & 6.02 & 7.4 & 80 & 17.2 & 7.75 & 150 & 32.25 \\
\hline $\mathrm{EC}$ & 298.74 & 99.58 & 0.607438 & 205.33 & 68.44333 & 0.417504 & 195.5 & 65.16667 & 0.397517 \\
\hline TDS & 182.74 & 36.548 & 0.133766 & 147.58 & 29.516 & 0.108029 & 128.48 & 25.696 & 0.094047 \\
\hline TSS & 125 & 25 & 0.0915 & 215 & 43 & 0.15738 & 320 & 64 & 0.23424 \\
\hline TH & 113.5 & 37.83333 & 0.230783 & 73.4 & 24.46667 & 0.149247 & 83.2 & 27.73333 & 0.169173 \\
\hline chloride & 37.9858 & 15.19432 & 0.111222 & 23.28 & 9.312 & 0.068164 & 32.82 & 13.128 & 0.096097 \\
\hline DO & 7.68 & 72.08333 & 26.3825 & 8.9 & 59.375 & 21.73125 & 8.2 & 66.66667 & 24.4 \\
\hline BOD & 6.84 & 136.8 & 50.0688 & 3.8 & 76 & 27.816 & 4.5 & 90 & 32.94 \\
\hline sulphate & 9.64 & 6.426667 & 0.078405 & 6.84 & 4.56 & 0.055632 & 6.896 & 4.597333 & 0.056087 \\
\hline \multirow[t]{3}{*}{$\mathrm{TA}$} & 138.45 & 115.375 & 1.759469 & 102.34 & 85.28333 & 1.300571 & 89.5 & 74.58333 & 1.137396 \\
\hline & & $\sum$ WQI & 85.48388 & & $\sum$ WQI & 69.00378 & & $\sum \mathrm{WQI}$ & 91.77456 \\
\hline & & WQI & 85.37375 & & WQI & 68.91488 & & WQI & 91.65632 \\
\hline
\end{tabular}

TDS and TSS are to measure of total dissolved and suspended elements existing in a water and both

parameters must be within BIS standard limit of $500 \mathrm{mg} / \mathrm{l}$. Dissolved and suspended solid are contain both inorganic as well as organic in nature. The absorption of TDS for the stream water samples ranged from 182.74 to $448 \mathrm{mg} / \mathrm{l}$ during Pre-monsoon, from 91.58 to $152 \mathrm{mg} / \mathrm{l}$ during Monsoon and from 116.48 to $132.5 \mathrm{mg} / \mathrm{l}$ during Postmonsoon season, which were well within the BIS desirable limit of $500 \mathrm{mg} / \mathrm{l}$. Similarly, TSS values were also within the desirable limit with mean values of $190.42 \pm 120.266$ $\mathrm{mg} / \mathrm{l}, 222.69 \pm 131.52 \mathrm{mg} / \mathrm{l}$ and $278.76 \pm 106.88 \mathrm{mg} / \mathrm{l}$ during Pre-monsoon, Monsoon and Post-monsoon season, respectively...

Total Hardness (TH) can observed from the lather creating capacity of a stream water in that calcium and magnesium cations are mainly control the hardness. The experimental values of $\mathrm{TH}$ for the water samples of the Tungabhadra River during premonsoon, monsoon and post- 
monsoon season ranged from $134.6 \pm 29.73 \mathrm{mg} / \mathrm{l}, 72.3 \pm$ $10.2 \mathrm{mg} / \mathrm{l}$ and $96 \pm 41.23 \mathrm{mg} / \mathrm{l}$ one-to-one, and the values were within the BIS limit of $300 \mathrm{mg} / \mathrm{l}$. Grounded on the hardness values, Tungabhadra River water normally falls just below moderately hard to hard water group.

Chloride is one of the significant water quality constraint and is extensively circulated in landscape in the form of salts of potassium chloride $(\mathrm{KCl})$, calcium chloride $\left(\mathrm{CaCl}_{2}\right)$ and sodium chloride $(\mathrm{NaCl})$. Various sources contributing chloride in water are draining from different rocks by the way toward enduring, surface run-off from inorganic manures subordinate horticultural fields, water system release, creature nourishes, and so forth. Tungabhadra river which contain chloride during Pre-monsoon, Monsoon and Post-monsoon season were $45.23( \pm 7.7) \mathrm{mg} / \mathrm{l}, 29.8( \pm 7.3)$ $\mathrm{mg} / \mathrm{l}$ and $31.9( \pm 2.98) \mathrm{mg} / \mathrm{l}$, separately. In present-study the chloride, value are within under BIS desirable limit, i.e., 250 $\mathrm{mg} / \mathrm{l}$.

Table no .6 value of water quality index at Sampling station 3.

\begin{tabular}{lrrrrrrrrrr}
\hline & \multicolumn{3}{c}{ Pre-Monsoon } & \multicolumn{3}{c}{ Monsoon } & \multicolumn{3}{c}{ Post-Monsoon } \\
\hline Parameter & \multicolumn{1}{c}{ Vn } & Qn & \multicolumn{1}{c}{ Qn*Wn } & Vn & Qn & \multicolumn{2}{c}{ Qn*Wn } & Vn & Qn & \multicolumn{1}{c}{ Qn*Wn } \\
\hline pH & 6.7 & -60 & -12.9 & 6.8 & -40 & -8.6 & 6.85 & -30 & -6.45 \\
EC & 351.39 & 117.13 & 0.714493 & 260.52 & 86.84 & 0.529724 & 185.5 & 61.83333 & 0.377183 \\
TDS & 86.48 & 17.296 & 0.063303 & 152.38 & 30.476 & 0.111542 & 128.48 & 25.696 & 0.094047 \\
TSS & 121 & 24.2 & 0.088572 & 325 & 65 & 0.2379 & 325 & 65 & 0.2379 \\
TH & 142.89 & 47.63 & 0.290543 & 82.35 & 27.45 & 0.167445 & 72.5 & 24.16667 & 0.147417 \\
chloride & 45.355 & 18.142 & 0.132799 & 26.54 & 10.616 & 0.077709 & 28.5 & 11.4 & 0.083448 \\
D0 & 6.5 & 84.375 & 30.88125 & 8.1 & 67.70833 & 24.78125 & 7.95 & 69.27083 & 25.35313 \\
BOD & 3.5 & 70 & 25.62 & 2.7 & 54 & 19.764 & 2.6 & 52 & 19.032 \\
sulphate & 12.09 & 8.06 & 0.098332 & 14.6 & 9.733333 & 0.118747 & 6.896 & 4.597333 & 0.056087 \\
TA & 158.84 & 132.3667 & 2.018592 & 120 & 100 & 1.525 & 89.6 & 74.66667 & 1.138667 \\
& & $\sum W Q I$ & 47.00788 & & $\sum W Q I$ & 38.71332 & & $\sum W Q I$ & 40.06987 \\
& & WQI & 46.94732 & & WQI & 38.66344 & & WQI & 40.01825 \\
\hline
\end{tabular}

Table no .7 values of water quality index at Sampling station 4.

\begin{tabular}{lrrrrrrrrrr}
\hline & \multicolumn{3}{c}{ Pre-Monsoon } & \multicolumn{4}{c}{ Monsoon } & \multicolumn{3}{c}{ Post-Monsoon } \\
\hline Parameter & Vn & Qn & \multicolumn{1}{c}{ Qn*Wn } & Vn & Qn & Qn*Wn & Vn & Qn & \multicolumn{1}{c}{ Qn*Wn } \\
\hline pH & 8.12 & 224 & 48.16 & 7.65 & 130 & 27.95 & 7.85 & 170 & 36.55 \\
EC & 334.82 & 111.6067 & 0.680801 & 221.52 & 73.84 & 0.450424 & 220 & 73.33333 & 0.447333 \\
TDS & 182.74 & 36.548 & 0.133766 & 128.48 & 25.696 & 0.094047 & 195 & 39 & 0.14274 \\
TSS & 195 & 39 & 0.14274 & 85 & 17 & 0.06222 & 360 & 72 & 0.26352 \\
TH & 133.96 & 44.65333 & 0.272385 & 79.45 & 26.48333 & 0.161548 & 80.5 & 26.83333 & 0.163683 \\
chloride & 43.85 & 17.54 & 0.128393 & 32.82 & 13.128 & 0.096097 & 33.8 & 13.52 & 0.098966 \\
DO & 5.8 & 91.66667 & 33.55 & 7.9 & 69.79167 & 25.54375 & 6.3 & 86.45833 & 31.64375 \\
BOD & 2.84 & 56.8 & 20.7888 & 5.53 & 110.6 & 40.4796 & 3 & 60 & 21.96 \\
sulphate & 4.65 & 3.1 & 0.03782 & 7.18 & 4.786667 & 0.058397 & 7.1 & 4.733333 & 0.057747 \\
TA & 81.34 & 67.78333 & 1.033696 & 112.34 & 93.61667 & 1.427654 & 81.34 & 67.78333 & 1.033696 \\
& & EWQI & 104.9284 & & EWQI & 96.32374 & & EWQI & 92.36144 \\
& & WQI & 104.7932 & & WQI & 96.19964 & & WQI & 92.24244 \\
\hline
\end{tabular}

Table no .8 values of water quality index at Sampling station 5.

\begin{tabular}{|c|c|c|c|c|c|c|c|c|c|}
\hline \multirow[b]{2}{*}{ Parameter } & \multicolumn{3}{|c|}{ Pre-Monsoon } & \multicolumn{3}{|c|}{ Monsoon } & \multicolumn{3}{|c|}{ Post-Monsoon } \\
\hline & Vn & Qn & $\mathrm{Qn}^{*} \mathrm{Wn}_{\mathrm{n}}$ & Vn & Qn & $\mathrm{Qn}^{*} \mathrm{Wn}$ & $V_{n}$ & Qn & $Q_{n}^{*} W_{n}$ \\
\hline $\mathrm{pH}$ & 8.25 & 250 & 53.75 & 8.235 & 247 & 53.105 & 7.85 & 170 & 36.55 \\
\hline EC & 408.15 & 136.05 & 0.829905 & 268.13 & 89.37667 & 0.545198 & 285.6 & 95.2 & 0.58072 \\
\hline TDS & 247.89 & 49.578 & 0.181455 & 128.48 & 25.696 & 0.094047 & 133.5 & 26.7 & 0.097722 \\
\hline TSS & 385 & 77 & 0.28182 & 120 & 24 & 0.08784 & 326 & 65.2 & 0.238632 \\
\hline TH & 154.36 & 51.45333 & 0.313865 & 68.43 & 22.81 & 0.139141 & 180 & 60 & 0.366 \\
\hline chloride & 50.12 & 20.048 & 0.146751 & 39.5 & 15.8 & 0.115656 & 32.82 & 13.128 & 0.096097 \\
\hline DO & 5.1 & 98.95833 & 36.21875 & 6.32 & 86.25 & 31.5675 & 6.01 & 89.47917 & 32.74938 \\
\hline BOD & 8.6 & 172 & 62.952 & 6.1 & 122 & 44.652 & 8.1 & 162 & 59.292 \\
\hline sulphate & 15.6 & 10.4 & 0.12688 & 8.12 & 5.413333 & 0.066043 & 6.6 & 4.4 & 0.05368 \\
\hline \multirow[t]{3}{*}{$\mathrm{TA}$} & 164.12 & 136.7667 & 2.085692 & 119.35 & 99.45833 & 1.51674 & 81.34 & 67.78333 & 1.033696 \\
\hline & & $\sum$ WQI & 1568871 & & $\sum$ WQI & 131.8892 & & $\sum$ WQI & 131.0579 \\
\hline & & WQI & 156.685 & & WQI & 131.7192 & & WQI & 130.8891 \\
\hline
\end{tabular}

Table no .9 values of water quality index at Sampling station 6.

\begin{tabular}{|c|c|c|c|c|c|c|c|c|c|}
\hline \multicolumn{3}{|c|}{ Pre-Monsoon } & \multicolumn{3}{|c|}{ Monsoon } & \multicolumn{4}{|c|}{ Post-Monsoon } \\
\hline Parameter & Vn & Qn & $Q^{*} W_{n}$ & Vn & Qn & $Q_{n}{ }^{*} W n$ & Vn & Qn & $Q n^{*} W n$ \\
\hline $\mathrm{pH}$ & 7.88 & 176 & 37.84 & 7.65 & 130 & 27.95 & 8.2 & 240 & 51.6 \\
\hline EC & 397.78 & 132.5933 & 0.808819 & 240.35 & 80.11667 & 0.488712 & 235.6 & 78.53333 & 0.479053 \\
\hline TDS & 62.15 & 12.43 & 0.045494 & 140 & 28 & 0.10248 & 132 & 26.4 & 0.096624 \\
\hline TSS & 122 & 24.4 & 0.089304 & 365 & 73 & 0.26718 & 360 & 72 & 0.26352 \\
\hline TH & 160.33 & 53.44333 & 0.326004 & 61.5 & 20.5 & 0.12505 & 120.5 & 40.16667 & 0.245017 \\
\hline chloride & 52.75 & 21.1 & 0.154452 & 34.5 & 13.8 & 0.101016 & 35.7 & 14.28 & 0.10453 \\
\hline D0 & 6.5 & 84.375 & 30.88125 & 7.5 & 73.95833 & 27.06875 & 7.1 & 78.125 & 28.59375 \\
\hline BOD & 6.8 & 136 & 49.776 & 5.4 & 108 & 39.528 & 2.5 & 50 & 18.3 \\
\hline sulphate & 15.26 & 10.17333 & 0.124115 & 20.6 & 13.73333 & 0.167547 & 6.896 & 4.597333 & 0.056087 \\
\hline \multirow[t]{3}{*}{$\mathrm{TA}$} & 162.1 & 135.0833 & 2.060021 & 78.1 & 65.08333 & 0.992521 & 81.34 & 67.78333 & 1.033696 \\
\hline & & $\sum$ WQI & 122.1055 & & $\sum$ WQI & 96.79126 & & $\sum$ WQI & 100.7723 \\
\hline & & WOI & 121.9481 & & WOI & 96.66656 & & wol & 100.6424 \\
\hline
\end{tabular}

Table no .10 values of water quality index at Sampling station 7.

\begin{tabular}{|c|c|c|c|c|c|c|c|c|c|}
\hline \multirow[b]{2}{*}{ Parameter } & \multicolumn{3}{|c|}{ Pre-Monsoon } & \multicolumn{3}{|c|}{ Monsoon } & \multicolumn{3}{|c|}{ Post-Monsoon } \\
\hline & $\mathrm{Vn}_{\mathrm{n}}$ & $Q_{n}$ & $\mathrm{Qn}^{*} \mathrm{Wn}$ & Vn & $Q_{n}$ & $Q_{n}^{*} W n$ & Vn & Qn & $\mathrm{Q}^{\mathrm{n}} \mathrm{W} \mathrm{W}$ \\
\hline $\mathrm{pH}$ & 7.1 & 20 & 4.3 & 7.1 & 20 & 43 & 7.15 & 30 & 6.45 \\
\hline $\mathrm{EC}$ & 439.86 & 146.62 & 0.894382 & 212 & 70.66667 & 0.431067 & 218 & 72.66667 & 0.443267 \\
\hline IDS & 254.72 & 50.944 & 0.186455 & 120 & 24 & 0.08784 & 128.48 & 25.696 & 0.094047 \\
\hline TSS & 325 & 65 & 0.2379 & 370 & 74 & 0.27084 & 180 & 36 & 0.13176 \\
\hline TH & 157.77 & 52.59 & 0.320799 & 83.4 & 27.8 & 0.16958 & 68.5 & 22.83333 & 0.139283 \\
\hline chloride & 53.78 & 21.512 & 0.157468 & 33.5 & 13.4 & 0.098088 & 32.82 & 13.128 & 0.096097 \\
\hline DO & 8.1 & 67.70833 & 24.78125 & 9.1 & 57.29167 & 20.96875 & 8.05 & 68.22917 & 24.97188 \\
\hline BOD & 2.2 & 44 & 16.104 & 2.15 & 43 & 15.738 & 2.05 & 41 & 15.006 \\
\hline sulphate & 13.87 & 9.246667 & 0.112809 & 6.896 & 4.597333 & 0.056087 & 7.85 & 5.233333 & 0.063847 \\
\hline \multirow[t]{3}{*}{$\mathrm{TA}$} & 159.6 & 133 & 2.02825 & 81.34 & 67.78333 & 1.033696 & 169.5 & 141.25 & 2.154063 \\
\hline & & $\sum$ WQI & 49.12331 & & $\sum W Q I$ & 43.15395 & & $\sum$ WQI & 49.55024 \\
\hline & & WQI & 49.06003 & & WQI & 43.09835 & & WQI & 49.4864 \\
\hline
\end{tabular}

Chloride is one of the significant water quality constraint and is extensively circulated in landscape in the form of salts of potassium chloride $(\mathrm{KCl})$, calcium chloride $\left(\mathrm{CaCl}_{2}\right)$ and sodium chloride $(\mathrm{NaCl})$. Various sources contributing chloride in water are draining from different rocks by the way toward enduring, surface run-off from inorganic manures subordinate horticultural fields, water system release, creature nourishes, and so forth. Tungabhadra river which contain chloride during Pre-monsoon, Monsoon and Post-monsoon season were $45.23( \pm 7.7) \mathrm{mg} / \mathrm{l}, 29.8( \pm 7.3)$ $\mathrm{mg} / \mathrm{l}$ and $31.9( \pm 2.98) \mathrm{mg} / \mathrm{l}$, separately. In present-study the chloride, value are within under BIS desirable limit, i.e., 250 $\mathrm{mg} / \mathrm{l}$.

Total amount of oxygen dissolved in a water body is named as dissolved oxygen (DO) and its absorption depend on physical turbulence, chemical mixing and biological actions of the water body. Assessment of dissolved oxygen is very much important to mitigate from contamination. A dissolved oxygen concentration level of $4-6 \mathrm{mg} / \mathrm{l}$ is the range for a ideal water quality supporting aquatic life. DO level which comes under this ideal range is relied upon to be contaminated. The mean DO values ranged from a 6.384 $\pm 1.2 \mathrm{mg} / \mathrm{l}$ during pre-monsoon season, maximum DO observe in monsoon of $8.1322 \mathrm{mg} / \mathrm{l}( \pm 1.02)$. DO is minimum $(<4 \mathrm{mg} / \mathrm{l})$ at site $\mathrm{S} 5$ during Pre-monsoon, due to high industrial waste discharged directly into river stretch directly.

Over-all volume of dissolved oxygen essential by "aerobic microorganisms" for complete ruin of organic wastes existing in a water body is labelled as biochemical oxygen demand (BOD). Therefore, BOD is a pointer of natural contamination with higher number demonstrating 
more elevated amounts of natural contamination (Patel et al. 1983). BOD values specifically over $5 \mathrm{mg} / \mathrm{l}$ are objectionable and the present examination discovered the mean BOD as $4.78( \pm 2.57) \mathrm{mg} / \mathrm{l}, 3.96( \pm 1.7) \mathrm{mg} / \mathrm{l}$ and 3.6 $( \pm 2.136) \mathrm{mg} / \mathrm{l}$ amid pre-monsoon, monsoon and postmonsoon season, respectively. The higher estimations of BOD underscored the nearness of conspicuous natural contamination source close to the sampling station.

Presence of sulphate in stream water is for the most part normal in nature contributed essentially by mineral sources like gypsum, and so on. Despite the fact that in little fixation sulphate is innocuous, in any case, high convergence of sulphate in drinking water may cause different intestinal infections. Mean sulphate convergence of the water tests under scrutiny fluctuated from $11.134 \mathrm{mg} / \mathrm{l}$ $( \pm 4.23)$ amid pre-monsoon season to $6.8 \mathrm{mg} / \mathrm{l}( \pm 0.76)$ during post-monsoon season and the qualities were within. the desirable limits of $150 \mathrm{mg} / \mathrm{l}$ according to BIS.

Total alkalinity is the ability of an aqueous solution to defuse an acid. Alkalinity is formed because of the different bicarbonate, carbonate and hydroxide particles present in water. The average absorption of alkalinity present in water samples was observed to be $142.85( \pm 29.5)$ $\mathrm{mg} / \mathrm{l}, 96.83( \pm 22.2) \mathrm{mg} / \mathrm{l}$ and $92.8( \pm 35.3) \mathrm{mg} / \mathrm{l}$ during premonsoon, monsoon and post monsoon season, respectively. The mean alkalinity

$\mathrm{WQI}_{\mathrm{a}}$ Analysis: The initial step in calculation of $\mathrm{WQI}_{\mathrm{a}}$ using "weighted arithmetic index" method is the assessment of unit weight of each physico-chemical constraints well thought-out for the investigation. The unit weight assigning process is done to transform the constraints of different scale to a common scale. As an example, unit weights and the drinking quality standards assigned to each constraint for WQI $_{a}$ is shown in Table 3. Higher the weights assigned higher is their significance in calculating $\mathrm{WQI}_{\mathrm{a}}$. Maximum unit weight, i.e., $36.6 \%$ is allocated to both BOD and DO, along these lines proposing the key essentialness of these couple constraints in water quality evaluation and their significant effect on the $\mathrm{WQI}_{\mathrm{a}}$. Tables 4 to 10 experiential values of the all the physico-chemical parameters (10 parameters are selected) and their $\mathrm{WQI}_{\mathrm{a}}$ from all the inspecting sites for three seasons under consideration. Of all the selected parameters DO and BOD had significant influence on the $\mathrm{WQI}_{\mathrm{a}}$ values as presented in tables 4 to 10 .

Table 11 summarizes the $\mathrm{WQI}_{\mathrm{a}}$ values from all stretch for each season. Most of the samples sandwiched between good to unsuitable water category $(41<\mathrm{WQI}<157)$. Premonsoon season recorded highest $\mathrm{WQI}_{\mathrm{a}}$ values ranging between 41.7 at site SS6 to 156.7 at site SS5 with an average WQI value of $112.47 \pm 30.02$ (Table 11). Direct discharge from nearby urban residents and industries resulted in the poor river quality further exacerbated by low flow in the pre-monsoon season.

Yamakanamardi and Sebastian (2013) reported similar results for the Cauvery River. Current research revealed that the most polluted sites are SS4, SS5and site SS6 based on the $\mathrm{WQI}_{\mathrm{a}}$ values along the entire reach of the Tungabhadra River. The WQI value of site SS5 indicate that the water quality is unfit for use be it; drinking, aquaculture, recreation or irrigation (Table 1). Rehana and Mujumdar (2011) in their paper also presented the analogous water quality for Tunga-Bhadra River. In the same way, inspecting site SS4, i.e., Nalavagalu and kumarapatanam town, the overpopulated municipal cluster laterally Tungabhadra River also observed a extremely degraded water quality primarily contributed by massive demographic over and above socio-economic burden in the form of stream bed infringement and stream water misuse for numerous chores.

The, site SS5 held very poor to notfit water quality status as indicated by the WQI values of 156.7 during postmonsoon season and 131.89 during monsoon season (Table no.11). In this manner, sampling station SS5 imprisoned extremely poor to unfit water quality trend as demonstrated by the $\mathrm{WQI}_{\mathrm{a}}$ estimations of 156.7 in the period of post-rainy season and 131.89 amid rainy season (Table no.11). Correspondingly, the poor water quality at sampling sites SS4, SS5 and SS6 is the consequence of direct discharge of sewage from adjoining urban settlements viz. Nalawagalu town, Nadiharahali and Airani, respectively.

Table no.11 Summary of water quality index of Tungabhadra stream.

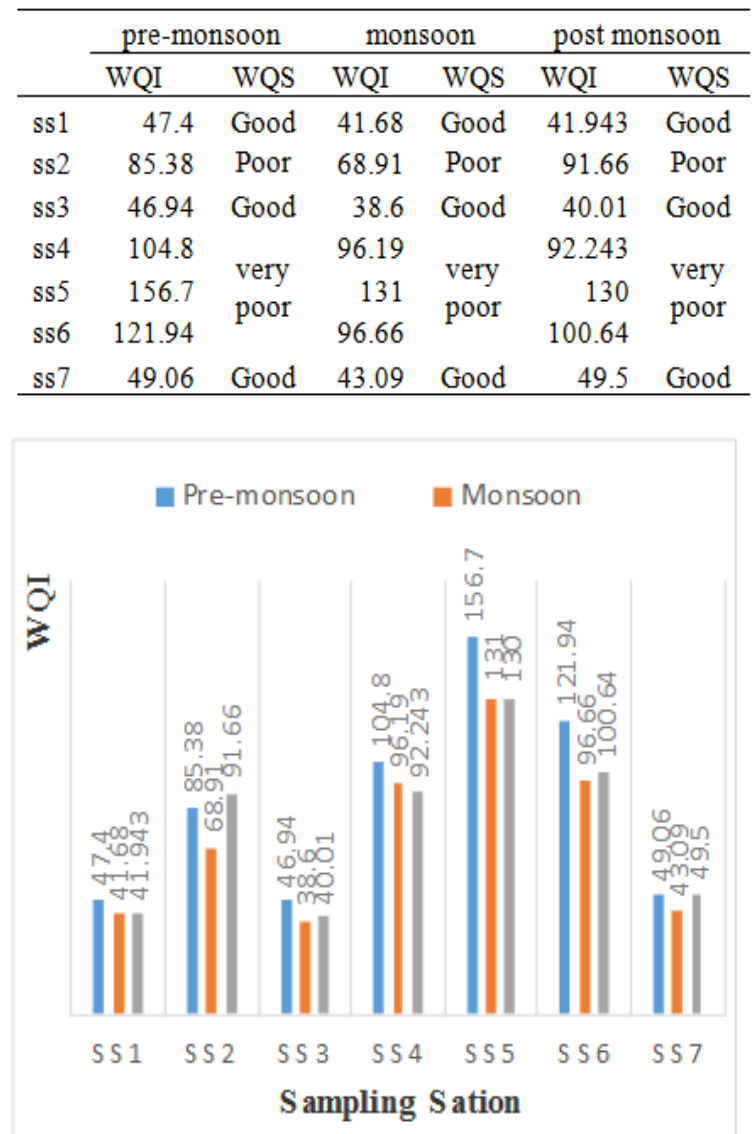

Fig.2 Water quality index rating of various sampling sites of Tungabhadra river

The poor water quality at these sites as indicated by $\mathrm{WQI}_{\mathrm{a}}$ values can be traced back to the anthropogenic activities such as leaking/ improperly managed sanitation facilities, direct sewage discharge and solid waste dumping, industrial discharge, etc. from Tables 4 to 10 clearly signifies BOD

Blue Eyes Intelligence Engineering 
and DO were the most influencing parameters in $\mathrm{WQI}_{\mathrm{a}}$ design. High organic pollution load contributed to high BOD concentration lowering the DO concentration along the stretch of Tungabhadra river as portrayed by water samples. The $\mathrm{WQI}_{\mathrm{a}}$ at sampling station SS6, i.e., close Airani, a rural area, was relatively good in comparison to all the deliberate locations with values wavering from 100 during winter days to 96.5 during rainy period. Moving further downstream along the stretch site SS7 hosted comparatively enhanced water quality owing to the dilution of pollution, microbial degradation of the organic load and scanty residents along the river. The pollution levels indicated seasonal change in water quality that is improved water quality during monsoon compared to pre and post monsoon (fig 2). As seen in Figure 2 there is a gradual decrease in pollution level moving downstream from SS5 to SS7. The trough in the graph at station 3 indicate the remote location of the site without much anthropogenic influence. However, contrasting high pollution load in station SS2 and SS4 can be related to demographic as well as industrial raw wastewater discharge. In all season, the water qualities were found to be good for the stations s1, SS3, and SS7 herein after referred as fit-sites while poor to unsuitable at site $\mathrm{SS} 2$, SS4, S5 and SS6 herein after referred as unfit-sites as depicted in Fig.2. During pre-monsoon season and postmonsoon, water quality of the sampling sites was found to fall under unsuitable to poor water quality attributable to the low flow in the river and the direct discharge of organic load at the unfit-sites. In contrast to this, the monsoon season showed improvement in water quality for all sampling stations indicating the dilution of pollutants through increased waterflow. Despite the increased flow during monsoon, the $\mathrm{WQI}_{\mathrm{a}}$ score at site SS2, SS4, SS5 and SS6 showed unsuitable water quality rating throughout year for the reason that of the flow is insufficient to increased organic pollution load, thus reducing the self-purification capacity of the river at these sites.

\section{CONCLUSION}

$\mathrm{WQI}_{\mathrm{a}}$ stands out to be a very useful approach in valuation and management of water quality. This study is first of its kind undertaken on the Tungabhadra river stretch in Karnataka. provides valuable vision into the status of overall relevance of a studied-on Tungabhadra river stretch constructed on $\mathrm{WQI}_{\mathrm{a}}$ values. It also provides a profounder thoughtful of communication of many physico-chemical constraints upon the over-all water-quality of a Tungabhadra river. The seasonal variation in the water quality of the Tungabhadra river based on the on the analysis of water samples from seven different sampling stations was carried out in this study. The study has both academic value and practical significance. Based on experiential $\mathrm{WQI}_{\mathrm{a}}$ values it can be determined that powerful action measures are immediately required to recover the Tungabhadra river water quality. It also suggests immediate need for appropriate water quality management plan that addresses any impending strategy for justifiable stream rebuilding. Appropriate measures could take up towards restriction of discharge of raw industrial and sewerage from residential/commercial establishments, storm-water channel into the stream and avoiding unabated throwing away of solid waste by societies living along the stream side. Furthermore, desilting actions to recover the conveying capacity of the stream channel needs to be adopted. Any activities of development involving encroachment of river should be strictly prohibited along the river corridor.

\section{REFERENCES}

1. Akkaraboyina M, Raju B (2012) A Comparative Study of Water Quality Indices of River Godavari. Int J Eng Res Dev 2(3):29-34.

2. Avvanavar SM, Shrihari S (2008) Evaluation of water quality index for drinking purposes for river Netravathi. Environ Monit Assess 143:279-290.

3. Bhargava DS (1983) Use of a water quality index for river classification and zoning of the Ganga River. Environ Pollut (Ser B) 6:51-67.

4. Bhargava DS, Saxena BS, Dewakar A (1998) A study of geopollutants in the Godavary river basin in India, Asian Environment. IOS Press, Amsterdam, pp 36-59.

5. Bharti N, Katyal D (2011) Water quality indices used for surface water vulnerability assessment. Int J Environ Sci 2(1):154-173.

6. Brown RM, McClelland NI, Deininger RA, Tozer RG (1970) Water quality index-do we dare? Water Sew Works 117(10):339-343.

7. Brown RM, McClelland NI, Deininger RA, O'Connor MF (1972) A water quality index-crashing the psychological barrier. In: Indicators of environmental quality Burden FR, Mc Kelvie I, Forstner U, Guenther A (2002) Environmental monitoring handbook. Mc grawHill Handbooks, New York, pp 3.1-3.21.

8. CPCB, ADSORBS/3 1978-1979) Scheme for zoning and classification of Indian Rivers: estuaries and coastal waters. CPCB website: www.CPCB.nic.in

9. Dalkey NC (1968) DELPHI. The Rand Corporation, Santa Monica Dalkey NC, Helmer O (1963) An experimental application of the Delphi method to the use of experts. Manag Sci 9(3):458-467.

10. De AK (2003) Environmental chemistry, 5th edn. New Age International Publisher, New Delhi, pp 190, 215, 242-244 Dee N, Baker J, Drobny N, Duke K, Whitman I, Fahringer D (1973).

11. An environmental evaluation system for water resource planning. Water Resour Res 9(3):523-535 DEQ (2003) The Oregon Department of Environmental Quality.

12. Dinius SH (1987) Design of an index of water quality. WaterRes Bull23(5):833-843

13. Dojlido J, Raniszewski J, Woyciechowska J (1994) Water quality index-application for river in Vistula River Basin in Poland. Water Sci Technol 30(10):57-64

14. Dwivedi S, Tiwari IC, Bhargava DS (1997) Water quality of the riverGanga at Varanasi. Inst Eng Kolkata 78:1-4

15. Helmer O, Rescher N (1959) On the epistemology of the inexact science. Manag Sci 6:25-53

16. Horton RK (1965) An index number system for rating ater quality. J Water Pollut Control Fed 37(3):300-306

17. Indian Standard Specification for Drinking Water (1983) IS-10500- 1983. Indian Standards Institution, New Delhi, Gr 6

18. Jayaprakash RI (1988), A study of the environmental biology of Netravathi river system. Thesis (Ph.D.) Mangalore University, pp 1-7, 9-14, 16-20, 25-27, 3032, 106-107,113-114. 
19. Khan F, Husain T, Lumb A (2003) Water quality evaluation and trend analysis in selected watersheds of the atlantic region of Canada. Environ Monit Assess 88(1):221-248.

20. Landwehr JM, Deininger RA (1976) A comparison of several waterquality indices. J Water Pollut Control Fed 48(5):954-958.

21. Leo ML, Dekkar M (2000) Hand book of water analysis (1-25,115-117, 143, 175, 223-226, 261, 273, 767).

22. Marcel Dekker, New York Lumb A, Halliwell D, Sharma $\mathrm{T}$ (2006) Application of CCME water quality index to monitor water quality: a case of the Mackenzie River basin, Canada. Environ Monit Assess 113:411-429.

23. McClelland NI (1974) Water quality index application in the Kansas River Basin. EPA-907/9-74-001. US EPA Region VII. Kansas City, MO Metcalf, Eddy (eds) (1972) Wastewater engineering: collection, treatment and disposal. McGraw Hill, New York, p 740.

24. Ott WR (1978) Environmental indices: theory and practice. Ann Arbor Science Publishers, Ann ArborSawyer CN, Mc Carthy PL, Parkin GF (1994) Chemistry for environmental engineering, 4th edn. Mc Graw-Hill International Edition, New York, pp 365-577

25. Smith RA, Alexander RB, Wolman MG (1987a), Analysis and interpretation of water-quality trends in major U.S. rivers, 1974-81. U.S. Geological Survey Water-Supply Paper 2307.

26. Smith RA, Alexander RB, Wolman MG (1987b) Waterquality trends in the Nation's rivers. Science 235:16071615.

27. Sobhani N (2003) The review on water quality index methods and their applications on Zoning of Karoon River. Thesis (M.Sc), Environmental Faculty, Science and Industry University.

28. Sebastian J, Yamakanamardi SM (2013) Assessment of water quality index of Cauvery and Kapila Rivers and at their confluence. Int J Lakes Rivers 6(1):59-67.

29. Tiwari TN, Mishra M (1985) A preliminary assignment of water quality index to major rivers. Ind $\mathrm{J}$ Environ Protect 5:276.

30. Train RE (1979) Quality Criteria for Water. U.S. Environmental Protection Agency, Washington, DC, pp $16,17,109$.

31. Tyagi S, Sharma B, Singh P, Dobhal R (2013) Water quality assessment in terms of water quality index. Am J Water Resour 1(3):34-38 US EPA (2009). Environmental impact and benefits assessment for final effluent guidelines and standards for the construction and development category. Office of Water, Washington, DC. EPA- 821-R-09-012.

32. Walsh P, Wheeler W (2012) Water quality index aggregation and cost benefit analysis. U.S. Environmental Protection Agency, Working Paper, 1205 .

33. Walski TM, Parker FL (1974) Consumer's water quality index. J Environ Eng ASCE 100:593-61. 